\title{
Effect of homogenization and ultrasonication on the physical properties of insoluble wheat bran fibres**
}

\author{
Ran Hü ${ }^{1}$, Min Zhang ${ }^{1 *}$, Benu Adhikari ${ }^{2}$, and Yaping Liu $^{3}$ \\ ${ }^{1}$ State Key Laboratory of Food Science and Technology, School of Food Science and Technology, Jiangnan University, \\ 214122 Wuxi, Jiangsu, China \\ ${ }^{2}$ School of Applied Sciences, RMIT University, City Campus, Melbourne, VIC 3001, Australia \\ ${ }^{3}$ Guangdong Galore Food Co. Ltd, Zhongshan 528447, China \\ Received February 4, 2015; accepted August 28, 2015
}

\begin{abstract}
A b s t r a c t. Wheat bran is rich in dietary fibre and its annual output is abundant, but underutilized. Insoluble dietary fibre often influences food quality negatively; therefore, how to improve the physical and chemical properties of insoluble dietary fibre of wheat bran for post processing is a challenge. Insoluble dietary fibre was obtained from wheat bran and micronized using high-pressure homogenization, high-intensity sonication, and a combination of these two methods. The high-pressure homogenization and high-pressure homogenization+high-intensity sonication treatments significantly $(\mathrm{p}<0.05)$ improved the solubility, swelling, water-holding, oil-holding, and cation exchange capacities. The improvement of the above properties by highintensity sonication alone was marginal. In most cases, the highpressure homogenization process was as good as the high-pressure homogenization+high-intensity sonication process in improving the above-mentioned properties; hence, the contribution of highintensity sonication in the high-pressure homogenization+highintensity sonication process was minimal. The best results show that the minimum particle size of wheat bran can reach $9 \mu \mathrm{m}$, and the solubility, swelling, water-holding, oil-holding, cation exchange capacities change significantly.

$\mathrm{K}$ e y w o r d s: homogenization, ultrasonication, wheat bran, insoluble dietary fibre, physical properties
\end{abstract}

\section{INTRODUCTION}

Wheat bran (WB) is a nutritionally valuable albeit underutilized by-product of wheat milling industry. It accounts for about $25 \%$ of the original grain weight (Neves et al., 2006). If all the available wheat around the world were milled to be consumed as food, the wheat bran as a by-product would account for approximately 15 million

\footnotetext{
*Corresponding author e-mail: min@jiangnan.edu.cn

**This work was financially supported by the Guangdong Province R and D Project No. 2012B091000125 (2012-2015).
}

tons per year (Prückler et al., 2014). It is rich in unsaturated fatty acids and dietary fibre (DF). Wheat bran contains about 36.5-52.4\% total dietary fibre DF (TDF) out of which $35.0-48.4 \%$ is insoluble DF (IDF) and $1.5-4.0 \%$ is soluble DF (SDF) (Vitaglione et al., 2008). The chemical composition of wheat bran fibres is arabinoxylan (accounted for $55 \%$ ), cellulose, lignin, $\beta$-glucan, and fructan (Cornell and Hoveling, 1998; Kamal-Eldin et al., 2009). Thus, wheat bran is a promising source of dietary fibre. A large number of scientific reports have shown that fibre-rich diets are important for maintaining good health and preventing chronic diseases. For this reason, the World Health Organization (WHO) recommended that the minimum daily intake of dietary fibre should be $12.5 \mathrm{~g} / 1000 \mathrm{kcal}$ (Heaney, 2006), based on energy intake. Currently, the average worldwide consumption of DF is lower than the recommended level; hence, increased incorporation of IDF in food products is necessary (Zhu et al., 2014). The rough mouthfeel and gritty texture associated with wheat bran are major limitations of increased incorporation of IDF in food products. The inclusion of IDF in most food products gives rise to the undesirable sensory changes, especially in colour and texture (Foschia et al., 2013). Thus, research is being undertaken in many laboratories to find alternative treatment to improve the quality of IDF so that it can be incorporated in food materials without compromising consumer appeal. The particle size of IDF (mainly cellulose) is reduced before incorporating it in several fibre-rich plant matrices (Hemery et al., 2011). The reduction of particle size alters the structure, surface area, as well as functional properties of the IDF.

(C) 2015 Institute of Agrophysics, Polish Academy of Sciences 
Mechanical, chemical, enzymatic, and other hybrid treatment methods have been used to obtain cellulose micro/ nanofibres from cellulosic materials. The mechanical method includes grinding (Ifuku et al., 2013), cryomilling (Alemdar and Sain, 2008), high-pressure homogenization (HPH) (Wang etal., 2014), and high-intensity ultrasonication (HIU) (Lu etal., 2013). The chemical and enzymatic methods include acid hydrolysis (Gliozzi et al., 2014) and enzymeassisted hydrolysis (Hassan et al., 2014), respectively. A number of hybrid methods have also been applied including 2,2,6,6-tetramethylpiperidine-1-oxyl radical (TEMPO)-mediated oxidation followed by mild mechanical treatment (Benhamou et al., 2014), synthetic and electrospinning methods (Cramariuc et al., 2013) etc. These methods lead to the formation of different micro/nanofibrillar cellulose, depending on the raw material and the pretreatment method used. High-pressure homogenization (HPH) is an economic and efficient processing technology to produce cellulose micro/nano fibres. During the HPH process, the aqueous suspension of fibres experiences high pressure, high shear, turbulence, and cavitation caused by rapid changes in pressure when the material passes through the homogenization chamber (Lee et al., 2009; Wang et al., 2012a). The combination of high pressure, high shear, turbulence, and cavitation during the HPH process disintegrates the amorphous regions and reduces the size of cellulose fibres. High-intensity ultrasonication (HIU) is suggested to be a good method for isolating micro/nanofibres that are relatively longer (Chen et al., 2011 a, 2011b, 2011c). Ultrasound energy is transferred to cellulose chains through the cavitation process, which involves the formation, growth, and violent collapse of microbubbles in water. The energy provided by cavitation is approximately $10-100 \mathrm{~kJ}$ $\mathrm{mol}^{-1}$, which include most energy of a hydrogen bond (Tischer et al., 2010). During the ultrasound treatment, the asymmetric interface between cellulose and water would be deformed by the bubbles produced by cavitation. When the rapidly forming and collapsing microbubbles interact with and impact on the cellulose surface, they erode and denude the surface, which leads to fibrillation and formation of nanofibres (Chen et al., 2011b).

Further research on improving the processes used to produce submicron-sized powders from dietary fibres is important and anticipated. However, a potentially useful hybrid method that combines high-pressure homogenization and ultrasound has not been applied to produce cellulose nanofibers, especially from wheat bran. Hence, the objective of this study was to evaluate and compare the effects of the micronization process involving high pressure homogenization, high-intensity ultrasonication, and a combination of the two methods on the particle size, characteristics, and physical properties (solubility, swelling, water-holding, oil-holding, and ion-exchange) of IDF prepared from wheat bran. The effect of the initial concentration of IDF on the above-mentioned properties of micronized IDF was also studied. We show that the micronized product can be preferably used in food formulations requiring high quality IDF.

\section{MATERIALS AND METHODS}

Insoluble dietary fibre was obtained from wheat bran using the procedure described previously (Zhu et al., 2010) with minormodification.Pulverized wheatbran(70-mesh)was defatted with hexane in a filter bag for $48 \mathrm{~h}$ at ambient temperature. The defatted bran was kept under a fume cupboard in order to remove the hexane by evaporation. $\mathrm{NaOH}$ was used to remove the protein from the defatted bran. The defatted sample $(100 \mathrm{~g})$ was mixed with $1000 \mathrm{ml}$ deionized water with continuous stirring and the temperature of this sample was maintained at $55^{\circ} \mathrm{C}$ for $2 \mathrm{~h}$ using a water bath. The $\mathrm{pH}$ of this defatted bran-water suspension was adjusted to 9.5 with a $5 \% \mathrm{NaOH}$ solution. The $\mathrm{pH}$-adjusted suspension was then centrifuged $(3000 \mathrm{~g}$ ) for $30 \mathrm{~min}$ and the residue was washed with distilled water several times until the supernatant was neutral. To remove the starch, the proteindevoid residue was suspended in distilled water $(500 \mathrm{ml})$ and $0.1 \mathrm{~g}$ of high temperature $\alpha$-amylase was added. The temperatureoftheamylase-containingsuspensionwasmaintained at $80^{\circ} \mathrm{C}$ with continuous/gentle agitation. Iodine staining was used to ascertain the absence of starch in the digested samples. The protein- and starch-devoid sample was bleached using an acidified sodium chlorite solution at $75^{\circ} \mathrm{C}$ for an hour (Li et al., 2014). The bleaching step was repeated three times. Finally, the residue was washed with cold water, lyophilized, and kept at $-30^{\circ} \mathrm{C}$ until used. The steps described above were repeated several times to obtain a sufficient amount of insoluble dietary fibre from the wheat bran.

The bleached wheat bran IDF was diluted to 0.2, 0.5, 0.8 , and $1.0 \%(\mathrm{w} / \mathrm{v})$ with distilled water. After the dilution, the suspension samples were roughly homogenized by an ultra-turrax (T18, IKA, German) at the max speed for $2 \mathrm{~min}$ intermittently. This step was carried out to avoid blockage during homogenization. Then, the suspension was passed through a high-pressure homogenizer (NS1001 L2K, Niro soavi, Italy) at 1000 bar. The sample was passed through the homogenizer 30 times. The time of homogenization was based on a preliminary experiment. Finally, the homogenized samples were lyophilized to obtain micronized IDF. The lyophilized samples were kept at $-30^{\circ} \mathrm{C}$ until used.

The bleached and freeze-dried samples were redispersed in distilled water at $0.2,0.5,0.8$, and $1.0 \%(\mathrm{w} / \mathrm{v})$ concentrations. These suspensions were homogenized roughly or preliminarily using the ultra-turrax at the max speed for $2 \mathrm{~min}$ intermittently. High-intensity ultrasound was applied to these suspensions immediately using a sonicator (JY-98 IIIN, Ningbo Xingzhi Technology Co., LTD, China). The sonicator $(1200 \mathrm{~W})$ was operated at $99 \%$ amplitude for $30 \mathrm{~min}$. This sonication time was used by Li et al. (2014) in a similar sonication study. The beaker containing the sample was partially immersed in an ice bath to ensure that the samples did not become overheated. The sonicated samples were freeze-dried and stored at $-30^{\circ} \mathrm{C}$ until use. 
In this method, the high-pressure homogenization step was followed by the high-intensity sonication. The samples obtained after the sequential homogenization and sonication were finally freeze-dried. The freeze-dried samples were stored at $-30^{\circ} \mathrm{C}$ until used.

The micron particle size and particle size distribution of the samples were measured with a laser scattering particle analyzer (Wang et al., 2012b) (S3500, Microtrac company, USA). The diameter, surface area per unit volume, D90, $\mathrm{D} 50$, and D10, which represent 90,50 , and $10 \%$ (on volume basis) of particles smaller than the size indicated, were determined. Each measurement was conducted in triplicate and the reported values are the mean values of these three runs. The IDF nanoparticle suspension samples were analysed using a Nanosizer (Malvern Instruments, UK) for particle size distribution ( Liu et al., 2014).

The morphology of the micronized IDF suspensions was studied using an optical microscope (OLYMPUS, BX41, Japan). In these tests, a drop of the suspension was placed on a glass slide and covered with a coverslip. All slides were observed in the bright-field transmission mode. The images were captured using a digital camera (OLYMPUS U-TV0.63XC, Tokyo, Japan) and the digitized images were processed using Adobe Photoshop CS5 (Adobe Systems Inc., CA).

The solubility of IDF after micronization was determined by stirring the sample in distilled water $(1: 20 \mathrm{w} / \mathrm{v})$ at ambient temperature for an hour. After centrifuging (3 $000 \times \mathrm{g}$ ) for $20 \mathrm{~min}$, the supernatant and residue were collected, oven-dried $\left(105^{\circ} \mathrm{C}\right)$, and weighed. The solubility was calculated as follows.

Solubility $(\%)=[$ weight of supernatant after drying $(\mathrm{g}) /$ weight of insoluble IDF $(\mathrm{g})] \times 100$.

Swelling capacity (SC) is defined as an increase in the volume of a known amount of fibre at a certain temperature when brought in contact with water or other solvents. $\mathrm{SC}$ was measured following the procedure reported in a previous report by Wang et al. (2013) with slight modification. The dry powder $(0.1 \mathrm{~g})$ was weighed in a $10 \mathrm{ml}$ $(0.1 \mathrm{ml}$ graduation) measuring cylinder and $10 \mathrm{ml}$ distilled water. The suspension was stirred gently with a glass rod to remove the trapped air. Then the cylinder was covered with preservative film (to prevent evaporation) and left undisturbed at room temperature overnight $(18 \mathrm{~h})$ for hydration. The volume $(\mathrm{ml})$ occupied by the sample was measured and $\mathrm{SC}$ was expressed as $\mathrm{ml} \mathrm{g}^{-1}$ of dry sample.

Water-holding capacity (WHC) was measured following the procedure reported earlier by (Hasnaoui et al., 2014). Twenty millilitres of distilled water were added to $0.1 \mathrm{~g}$ of lyophilized samples in a $50 \mathrm{ml}$ centrifuge tube. The sample was stirred and left at room temperature for $24 \mathrm{~h}$. After centrifuging at $3000 \times \mathrm{g}$ for $20 \mathrm{~min}$, the supernatants were carefully removed and the residues were weighed. The WHC was calculated as $g$ water/g dry sample.
Oil-holding capacity (OHC) was measured following procedures proposed in two previous studies (Wang et al., 2013) with minor modification. The procedure was the same as that used for measurement of water-holding capacity except for the use of commercial soybean oil instead of distilled water. The oil had a density of $0.917 \mathrm{~g} \mathrm{~m}^{-3}$ at $25^{\circ} \mathrm{C}$. $\mathrm{OHC}$ was expressed as g oil /g dry sample.

Cation-exchange capacity (CEC) was calculated according to the two methods reported previously (Wang et al., 2013). The IDF samples $(0.1 \mathrm{~g})$ were firstly acidified by adding $50 \mathrm{ml}$ of $0.01 \mathrm{~mol} \mathrm{l}^{-1}$ hydrochloric acid and gently stirred overnight at $4^{\circ} \mathrm{C}$. The acidified sample was centrifuged for $30 \mathrm{~min}$ at $3000 \times \mathrm{g}$. The residue was washed with deionized water until the $\mathrm{pH}$ of the supernatant was above 4.0. Then the washed acidic residue was suspended in $25 \mathrm{ml}$ of $0.3 \mathrm{~mol} \mathrm{l}^{-1}$ sodium chloride, stirred for $30 \mathrm{~min}$ at room temperature, and titrated with $0.01 \mathrm{~mol} \mathrm{l}^{-1}$ potassium hydroxide. The control (blank) test was performed in the same way using $50 \mathrm{ml}$ distilled water instead of hydrochloric acid. The CEC was expressed as milliequivalent per kilogram of dry sample (meq $\mathrm{kg} \mathrm{g}^{-1}$ dry sample).

All the experimental runs were conducted in triplicates. The mean value of the triplicate runs were reported for each determination. The significance of the differences between mean values was calculated at a $95 \%$ confidence level $(p<0.05)$. A SPSS 16.0 statistical package (IBM, Chicago, IL, USA) was used to conduct the statistical analysis.

\section{RESULTS AND DISCUSSION}

Table 1 shows the particle size distribution of wheat bran IDF subjected to high-pressure homogenization (HPH) and high-intensity ultrasonication (HIU) at different concentrations. When raw IDF samples were subjected to the combined HPH and HIU treatment, there was a significant decrease in particle size and an increase in surface area compared to their corresponding values in the HIU treatment. The HIU-treatment alone seemed to be inefficient in reducing the particle size of IDF.

The initial concentration seems to affect the micronization process. As shown in Table 1, the particle size of HIU, $\mathrm{HPH}$, and $\mathrm{HPH}+\mathrm{HIU}$ samples decreased with the increase in the initial IDF concentration. Clarke et al. (2010) have recently reviewed the causes of the breakup of particles in the high-pressure homogenization process and concluded that shear stress encountered by a fluid is the main cause of the particle breakup during HPH. An increase in the concentration of IDF increases the viscosity of the suspension (Elleuch et al., 2011), which increases the shear stress during HPH. An increase of viscosity affects turbulence due to the increase in pressure when the suspension passes through the homogenization valve (Engler and Robinson, 1981). However, the effect of the initial concentration on the IDF suspension is limited during HPH. As can be seen in Table 1, when the initial concentration varied from 0.2 to $1.0 \%$, the particle size and the surface area did not change significantly $(\mathrm{p}>0.05)$. 
T a b l e 1. Particle size distribution of wheat bran IDF after micronization at different initial solid concentrations

\begin{tabular}{|c|c|c|c|c|c|c|c|}
\hline $\begin{array}{c}\text { Initial } \\
\text { concentration } \\
(\%)\end{array}$ & Treatment & $\mathrm{MV}(\mu \mathrm{m})$ & $\mathrm{CS}\left(\mathrm{m}^{2} \mathrm{~cm}^{-3}\right)$ & $\mathrm{SD}(\mu \mathrm{m})$ & $\mathrm{D} 10(\mu \mathrm{m})$ & $\mathrm{D} 50(\mu \mathrm{m})$ & $\mathrm{D} 90(\mu \mathrm{m})$ \\
\hline & НPH & $13.56 \pm 0.32$ & $0.710 \pm 0.072$ & $7.70 \pm 0.04$ & $4.22 \pm 0.12$ & $11.6 \pm 0.16$ & $24.69 \pm 0.20$ \\
\hline \multirow[t]{3}{*}{0.2} & HPH+ HIU & $12.67 \pm 0.16$ & $0.825 \pm 0.005$ & $7.41 \pm 0.10$ & $3.57 \pm 0.04$ & $9.65 \pm 0.13$ & $23.85 \pm 0.17$ \\
\hline & $\mathrm{HIU}$ & $204.8 \pm 9.4$ & $0.048 \pm 0.003$ & $111.5 \pm 4.6$ & $62.60 \pm 7.31$ & $192.8 \pm 10.2$ & $349.5 \pm 9.0$ \\
\hline & $\mathrm{HPH}$ & $12.86 \pm 0.07$ & $0.749 \pm 0.007$ & $7.49 \pm 0.04$ & $4.00 \pm 0.04$ & $10.46 \pm 0.21$ & $24.09 \pm 0.18$ \\
\hline \multirow[t]{3}{*}{0.5} & HPH+ HIU & $11.03 \pm 0.40$ & $0.931 \pm 0.001$ & $6.68 \pm 0.09$ & $3.17 \pm 0.06$ & $8.55 \pm 0.08$ & $21.72 \pm 1.68$ \\
\hline & HIU & $180 \pm 8.3$ & $0.056 \pm 0.001$ & $100.5 \pm 5.3$ & $52.20 \pm 8.52$ & $183.6 \pm 8.8$ & $296.6 \pm 2.2$ \\
\hline & $\mathrm{HPH}$ & $12.44 \pm 0.36$ & $0.749 \pm 0.032$ & $6.76 \pm 0.11$ & $4.07 \pm 0.11$ & $10.75 \pm 0.33$ & $21.73 \pm 0.40$ \\
\hline \multirow[t]{3}{*}{0.8} & HPH+ HIU & $10.42 \pm 0.40$ & $1.004 \pm 0.009$ & $6.18 \pm 0.06$ & $2.93 \pm 0.01$ & $7.90 \pm 0.04$ & $19.86 \pm 1.24$ \\
\hline & HIU & $176.5 \pm 4.3$ & $0.053 \pm 0.003$ & $95.83 \pm 2.66$ & $56.57 \pm 2.14$ & $181.2 \pm 11.3$ & $288.3 \pm 4.3$ \\
\hline & $\mathrm{HPH}$ & $11.65 \pm 0.18$ & $0.804 \pm 0.016$ & $6.46 \pm 0.10$ & $3.80 \pm 0.07$ & $9.68 \pm 0.13$ & $20.9 \pm 0.40$ \\
\hline \multirow[t]{2}{*}{1.0} & $\mathrm{HPH}+\mathrm{HIU}$ & $9.77 \pm 0.06$ & $0.988 \pm 0.018$ & $5.89 \pm 0.11$ & $3.04 \pm 0.04$ & $8.09 \pm 0.06$ & $18.56 \pm 0.23$ \\
\hline & HIU & $179.2 \pm 3.7$ & $0.052 \pm 0.001$ & $96.46 \pm 3.73$ & $56.93 \pm 1.1$ & $181.2 \pm 7.2$ & $292.1 \pm 2.1$ \\
\hline
\end{tabular}

MV - mean diameter of the volume distribution, CS - calculated surface that provides an indication of the specific surface area, $\mathrm{SD}$ - standard deviation that is one measure of the width of the particle size distribution, D10, D50, D90 - 10, 50 and $90 \%$ of the volume that is smaller than the size indicated, respecitively.

When the IDF suspension was subjected to the HIUtreatment, the mean particle size decreased but was still larger than $170 \mu \mathrm{m}$ and the surface area was rather small (Table 1). The HIU treatment makes use of the cavitation effect of ultrasound to break down the aggregation of fibres while the axial shear force is weak (Chen et al., 2014), which leads to long cellulose fibres, resulting in larger size. The particle size of the homogenized suspension decreased with the increase in the initial concentration of IDF. However, when the concentration was increased to $1.0 \%$, the particle size started to decrease. This may be due to the fact that an IDF concentration of or above $1.0 \%$ increases the viscosity of the suspension and exerts greater resistance to the flow, which does not favour a further decrease in the particle size (Chen et al., 2012).

The particle size of the samples treated by $\mathrm{HPH}+\mathrm{HIU}$ sequentially decreased slightly and the specific surface area increased (Table 1). When the initial IDF concentration was $0.8 \%$, the specific surface area increased most. This may be due to the porous IDF structure after homogenization (Chau and Huang, 2003), which makes it easier to strip.
The particle size distribution and the presence and concentration of insoluble fibres have an appreciable effect on the physicochemical properties of dietary fibre (Gupta and Premavalli, 2010; Rosa et al., 2013). The decrease in the particle size was found to significantly improve the bioaccessibility and colonic metabolism of phenolic antioxidants in in vitro digestion studies (Hemery et al., 2010).

Figure 1 shows the light microscopic images of IDF suspensions subjected to the HPH, HIU, and HPH+HIU micronization treatments. We can see a large aggregate in Fig. $\mathrm{C}_{1}$, which is not involved in the HPH treatment. This observation suggests that HIU is not as effective as the HPH and can only be used as a complementary process to HPH micronization.

On the basis of water solubility, DF can be divided into soluble and insoluble fibres. As shown in Fig. 2, HPH and $\mathrm{HPH}+\mathrm{HIU}$ treated samples have higher solubility compared to the HIU ones. This figure also shows that the solubility decreases with an increase in the concentration, irrespective of the micronization methods used. Compared to the unmicronized samples, the solubility of all the micronized 

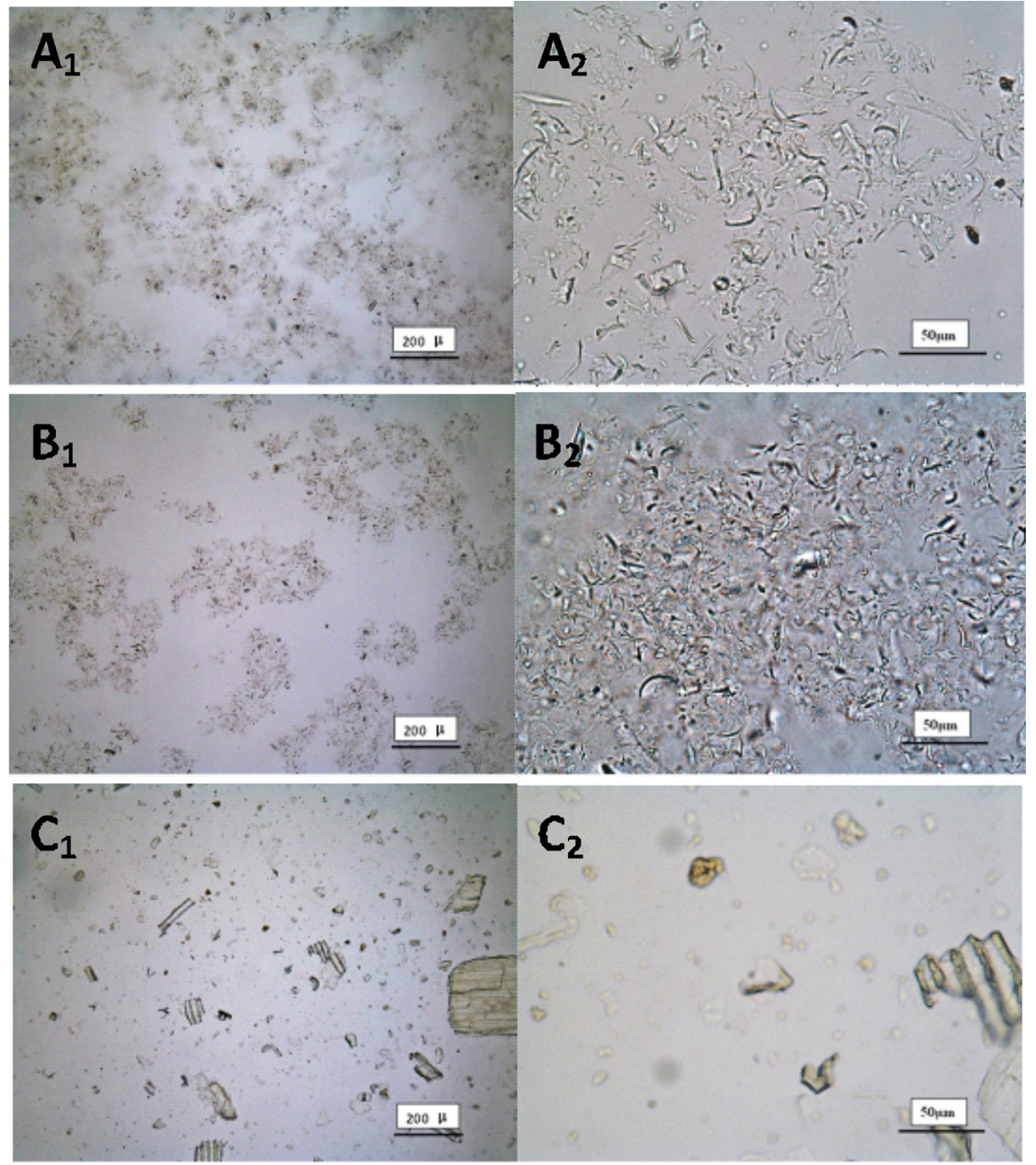

Fig. 1. Bright field microscopic images of the $0.5 \%$ micronized IDF sample. $A_{1} \times 100, A_{2} \times 500-H P H$ treated samples, $B_{1} \times 100, B_{2} \times 500$ $-\mathrm{HPH}+\mathrm{HIU}$ treated samples and $\mathrm{C}_{1} \times 100, \mathrm{C}_{2} \times 500-\mathrm{HIU}$ treated samples.

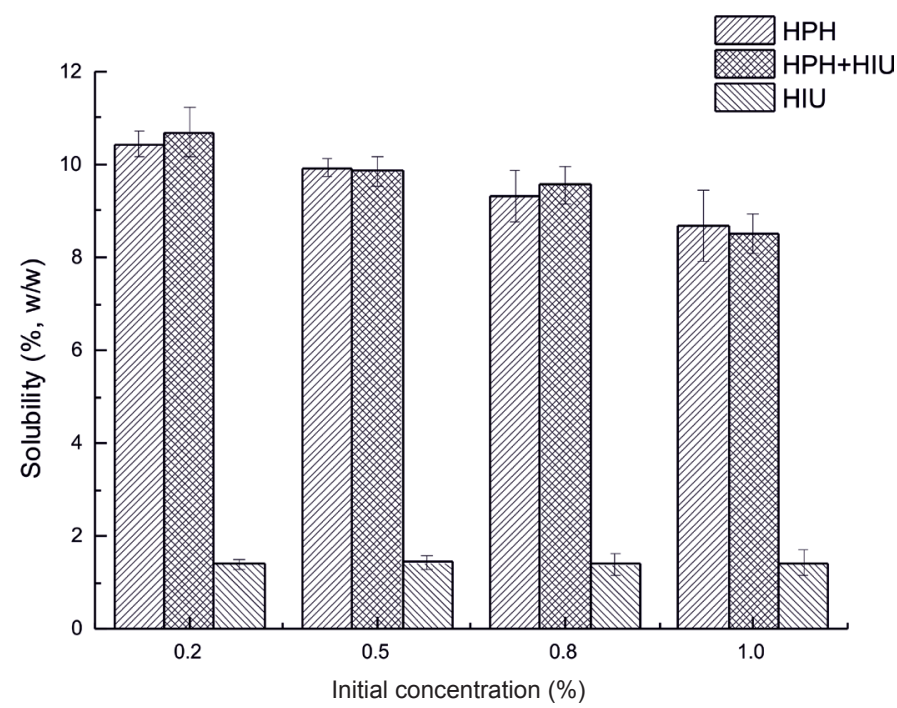

Fig. 2. Effect of micronization on the solubility of wheat bran IDF at different initial concentration. 


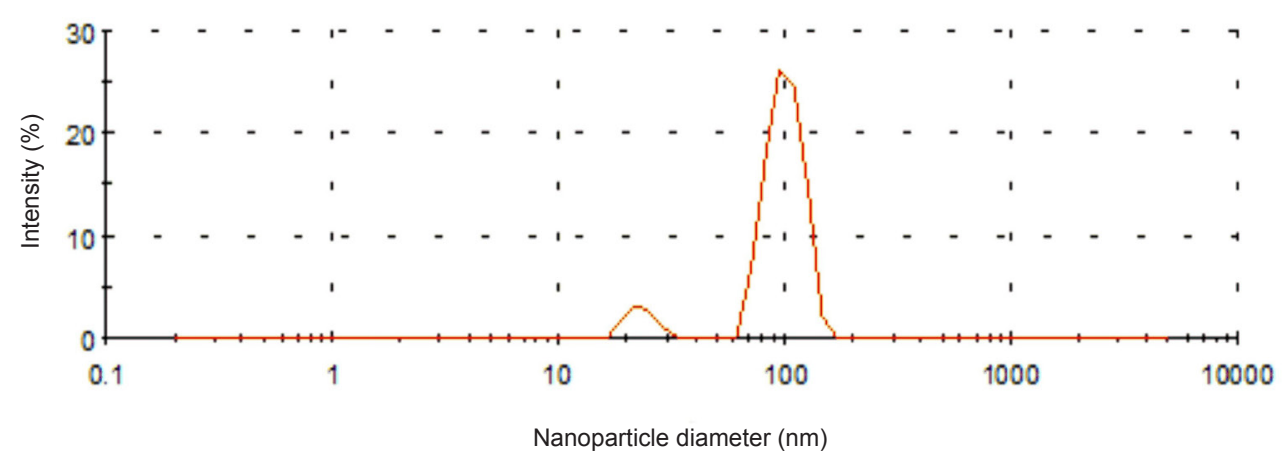

Fig. 3. Nanoparticle size distribution of a supernatant of the $0.2 \% \mathrm{HPH}$ sample after centrifugation at $4000 \mathrm{~g}$.

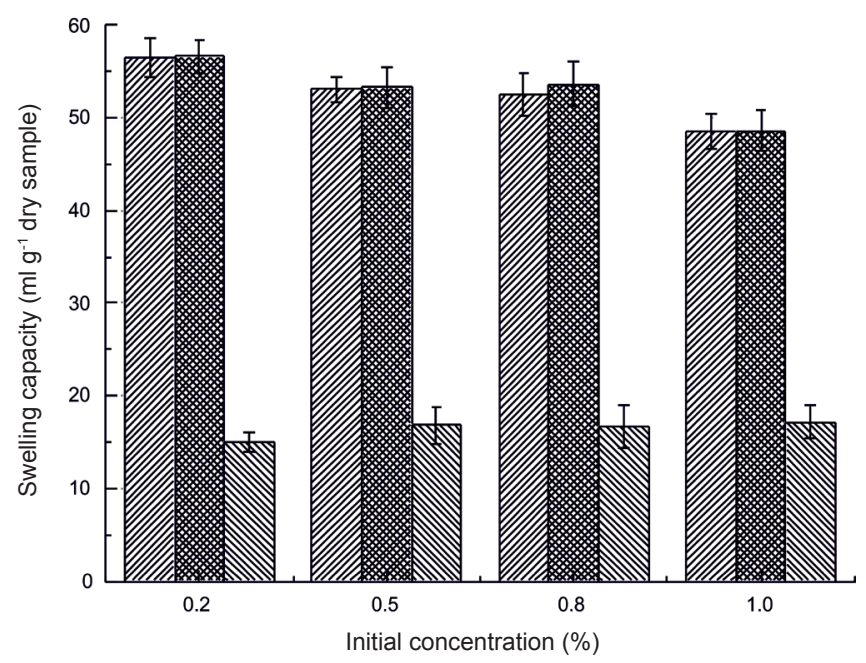

Fig. 4. Effect of micronization on swelling capacity of wheat bran IDF at different initial concentrations. Legend as on Fig. 2.

samples increased to some extent, suggesting that the application of homogenization and ultrasonication increased the soluble fraction of the dietary fibres (Chau et al., 2007). The increased solubility of the IDF was probably due to the increased surface area, decreased particle size, and the decrease in the cell-wall pectic substances caused by micronization (Chau et al., 2007). Some particles reaching the nanoscale are shown in Fig 3. Huang et al. (2010) reported that the small particle size caused by micronization might increase tremendously the porosity and capillary attraction of the fibre and consequently result in redistribution of the fibre composition from the insoluble to the soluble fraction.

The micronization reduced the particle size, increased the surface area, and altered the physical structure, which ultimately affected the swelling capacity (Fig. 4) and waterholding capacity (Fig. 5) of wheat bran IDF. The swelling capacity values of HIU treated samples were lower than $20 \mathrm{ml} \mathrm{g}^{-1}$ dry sample, which is much lower than the corresponding value of the HPH and HPH+HIU treated samples (Fig. 4). Moreover, the swelling capacity values of $\mathrm{HPH}+\mathrm{HIU}$ treated samples were almost equal to the HPH treated ones, most probably due to the similar particle size in these samples. A similar trend was observed in the case of water holding capacity (WHC) values in these samples. When the initial concentration of wheat bran IDF increased, the swelling capacity and WHC values of HPH and PHP+HIU treated samples decreased, which is consistent with the decrease in the particle size in these samples. This relationship observed between the particle size and swelling capacity and WHC is not in agreement with some earlier publications. For example, both Wang et al. (2012a, b) and Gupta and Premavalli (2010) found that the reduction in the particle size from resulted in increased hydration 


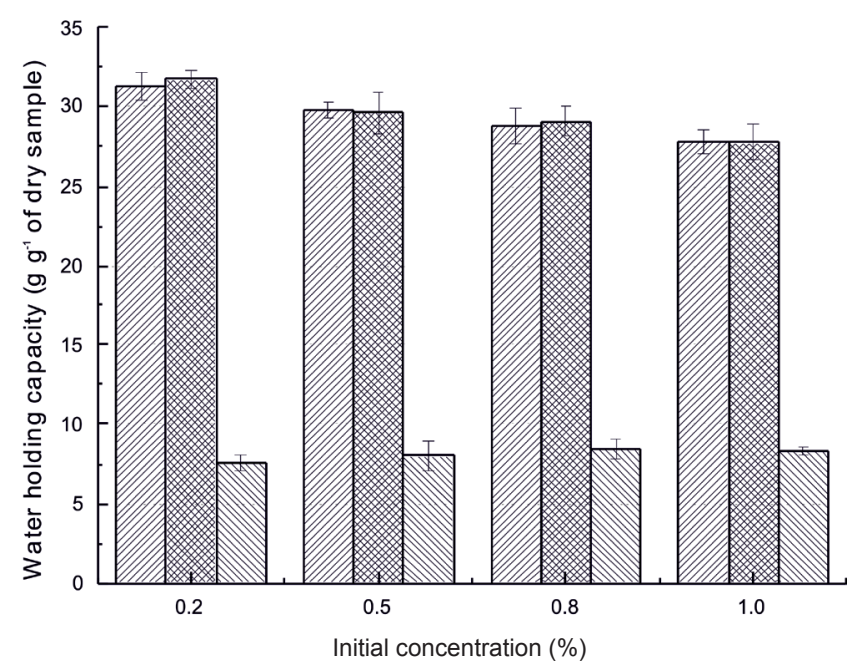

Fig. 5. Effect of micronization on the water holding capacity of wheat bran IDF at different initial concentrations. Legend as on Fig. 2.

properties. The contrasting observations in our result and that of the above-mentioned publications indicate that particle size is not the limiting factor that determines the swelling capacity and WHC of IDF. According to Huang et al. (2010), the small particle size caused by micronization substantially increases the porosity and capillary attraction of fibreand, consequently, increasesthesolublefraction. Chemical composition, alteration in physical structure, and aggregation brought about by the micronization methods also affect the hydration properties of IDF (Peng et al., 2011).

The water-binding ability of dietary fibre is generally related to its structure, density, and the nature and number of water-binding sites. It is then expected that the greatly reduced particle size (Table 1) caused by the HPH micronization or $\mathrm{HPH}+\mathrm{HIU}$ treatment would greatly increase the surface area and expose more water binding sites (eg polar groups or uronic acid groups) to the surrounding water (Chen et al., 2013), leading to significant improvement in the WHC and swelling (Chau et al., 2007). On the contrary, the shearing forces generated during HIU may cause the collapse of fibre matrix and pores, which could negatively affect the hydration properties of fibre materials.

It is worth mentioning here that irreversible agglomeration occurs during drying, which affects the particle size and ultimately influences the properties related to the particle size (Beck et al., 2012). The micronized IDF sample disperses well in water when the concentration is very low (less than $0.2 \%$ ). With the increase in the size and number of ice crystals during freezing, the IDF concentration increases due to the squeeze effect of ice crystals. During freeze-drying, the medium in which the IDF is dispersed is removed through sublimation. The formation of a hydrogen bond between the hydroxyl groups of adjacent cellulosic molecules of IDF get closer to each other until they assemble into elongated fibres and finally into a three-dimensional network structure.
However, when the concentration of IDF reached $0.5 \%$ or above, the close packing of the IDF in dispersion after micronization resulted in greater crosslinking and aggregation and formed a two-dimensional lamina-like structure during freeze drying (Chen et al., 2014; Heath and Thielemans, 2010). The HIU treated samples were less affected by the concentration due to their large particle size and smaller surface area.

The effect of micronization on the oil-holding capacity $(\mathrm{OHC})$ is illustrated in Fig. 6. As shown in this figure, the oil holding capacity $(\mathrm{OHC})$ of samples subjected to the HPH and $\mathrm{HPH}+\mathrm{HIU}$ treatments is almost the same $(\mathrm{p}>0.05)$ and approximately six times of the OHC of HIU treated ones. The OHC of samples treated with $\mathrm{HPH}$ and $\mathrm{HPH}+\mathrm{HIU}$ decreased when the initial concentration of IDF increased while the OHC values of HIU treated samples decreased with the increase in the IDF concentration.

Earlier studies have provided ambiguous reports regarding the relationship between particle size and $\mathrm{OHC}$ values of IDF. For example, Chen et al. (2013) reported that the $\mathrm{OHC}$ values increased with the decrease in particle size of IDF when subjected to high-pressure micronization process, while Gupta and Premavalli (Gupta and Premavalli, 2010) reported that the $\mathrm{OHC}$ value decreased with the decrease in particle size. Wang et al. (2009) suggested that variation of $\mathrm{OHC}$ with particle size is different when the processing method is different (wet milling, freeze-dried milling, and hot-air dried milling). Sangnark and Noomhorm (2003) reported that a decrease in the particle size of sugarcane bagasse resulted in a decrease in both the WHC and OHC. The above discrepancies are due to the use of different sources (and hence different chemical composition) and different size reduction methods in these studies (Chen et al., 2013). It is assumed that the decrease in particle size caused by high-pressure homogenization could increase the 


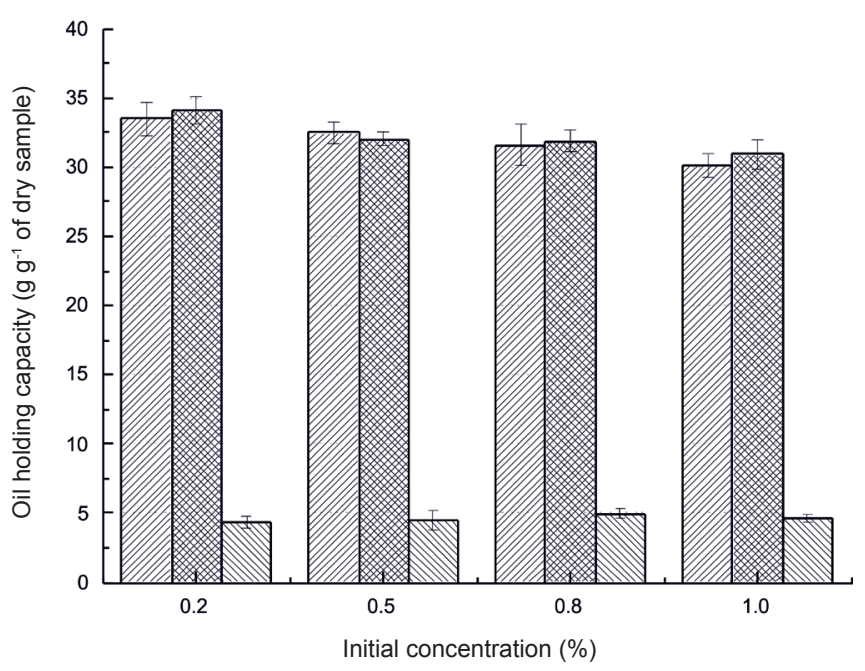

Fig. 6. Effect of micronization on the oil holding capacity $(\mathrm{OHC})$ of wheat bran IDF at different initial concentrations. Legend as on Fig. 2.

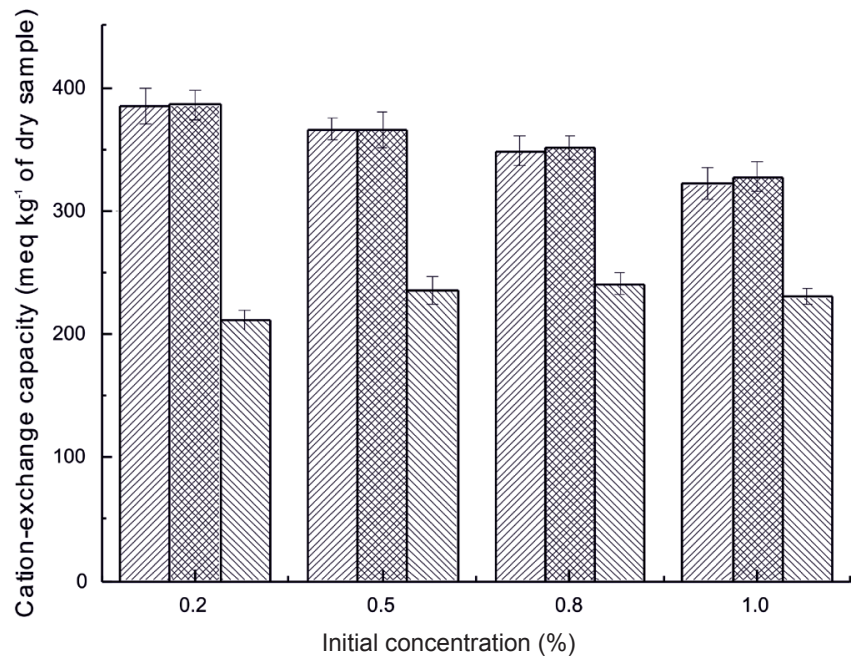

Fig. 7. Effect of micronization on cation-exchange capacity of wheat bran IDF with at different initial concentrations. Legend as on Fig. 2.

capillary attraction of fibres and, consequently, enhance the physical entrapment of oil, due to which the $\mathrm{OHC}$ increases (Huang et al., 2010).

The significant $(\mathrm{p}<0.05)$ increase in the specific surface area and some extent of a decrease in particle size of the $\mathrm{HPH}+\mathrm{HIU}$ treated sample compared to the HPH treated one did not significantly $(\mathrm{p}>0.05)$ affect the $\mathrm{OHC}$ values between these two samples. The OHC of HIU treated samples were significantly $(\mathrm{p}<0.05)$ lower than those of the $\mathrm{HPH}$ and $\mathrm{HPH}+\mathrm{HIU}$ treated samples. This may be caused by the fact that the HIU treatment causes collapse of fibre matrix and pores and reduces the particle size and surface area. The subsequent concentration during freezing and freeze-drying leads to an $\mathrm{OHC}$ decrease in IDF. The high $\mathrm{OHC}$ of the $\mathrm{HPH}$ and $\mathrm{HPH}+\mathrm{HIU}$ treated IDF suggests that
IDF produced in these processes can be used as a fibre-rich ingredient in food formulations requiring good oil retention and cholesterol absorption (Wang et al., 2012b).

Figure 7 shows the effect of HPH, HIU, and HPH+HIU on cation-exchange capacity (CEC). The CEC of the HPH and $\mathrm{HPH}+\mathrm{HIU}$ treated samples was between 300-400 meq $\mathrm{kg}^{-1}$ of dry sample, while the CEC values of a HIU samples were below 300 meq $\mathrm{kg}^{-1}$ of dry sample. The CEC values for the HPH and HPH+HIU samples were not statistically different $(\mathrm{p}>0.05)$ and decreased as the initial concentration IDF increased. The different IDF concentration did not seem to affect the CEC of HIU treated samples.

The increase in the CEC values with the decrease in the particle size can be attributed to the increased exposure of uronic acids or ion-binding sites (Chau et al., 2007). The 
increased surface area of the HPH and HPH+HIU treated samples increased the CEC compared to the HIU treated samples. The HPH and HPH+HIU process exposed a higher proportion of uronic acid moieties or cation-binding sites after the homogenization. The CEC values of $\mathrm{HPH}+\mathrm{HIU}$ and HPH treated samples were statistically similar $(\mathrm{p}>0.05)$ at a given concentration and decreased when the initial concentration increased. In the case of the HIU treated samples, the CEC value was not affected by the different initial concentration. This might be due to the aggregation of the fully micronized sample and the decreased specific surface area and binding sites.

The cation-exchange capability of IDFs can greatly influence their physiological and chemical functions. A higher CEC value indicates a stronger capability in reducing the number of intact micelles, the transit time, consequently reducing the total time available for the absorption of cholesterol in the small intestine (Chau et al., 2007).

\section{CONCLUSIONS}

1. High-pressure homogenization (HPH) alone and highintensity ultrasound (HIU) and HPH in combination can decrease the particle size of wheat bran insoluble dietary fibre. The HPH+HIU treatments increased the specific surface area of the particles. The HIU processing alone was not effective in reducing the particle size. The particle size of the IDF samples did not change significantly $(p>0.05)$ when HIU was applied after the HPH treatment, mainly due to its weak axial shear force.

2. The concentration of IDF in the dispersion was found to affect the solubility, swelling, water and oil holding and ion-exchange capacities through its effects on particle size. The HPH-treatment or the HPH+HIU treatment was found to greatly improve the solubility, water and oil holding and cation exchange capabilities of IDF, thereby making the resultant IDF suitable for food fortification.

\section{REFERENCES}

Alemdar A. and Sain M., 2008. Isolation and characterization of nanofibers from agricultural residues: wheat straw and soy hulls. Bioresour. Technol., 99, 1664-1671.

Beck S., Bouchard J., and Berry R., 2012. Dispersibility in water ofdriednanocrystalline cellulose. Biomacromolecules, 13, 1486-1494.

Benhamou K., Dufresne A., Magnin A., Mortha G., and Kaddami H., 2014. Control of size and viscoelastic properties of nanofibrillated cellulose from palm tree by varying the TEMPO-mediated oxidation time. Carbohydr. Polym., 99, 74-83.

Chau C.F. and Huang Y.L., 2003. Comparison of the chemical composition and physicochemical properties of different fibers prepared from the peel of Citrus sinensis L. Cv. Liucheng. J. Agric. Food Chem., 51, 2615-2618.

Chau C.F., Wang Y.T., and Wen Y.L., 2007. Different micronization methods significantly improve the functionality of carrot insoluble fibre. Food Chem., 100, 1402-1408.
Chen J., Gao D., Yang L., and Gao Y., 2013. Effect of microfluidization process on the functional properties of insoluble dietary fiber. Food Res. Int., 54, 1821-1827.

Chen P., Yu H., Liu Y., Chen W., Wang X., and Ouyang M., 2012. Concentration effects on the isolation and dynamic rheological behavior of cellulose nanofibers via ultrasonic processing. Cellulose, 20, 149-157.

Chen W., Li Q., Wang Y., Yi X., Zeng J., Yu H., Liu Y., and Li J., 2014. Comparative study of aerogels obtained from differently prepared nanocellulose fibers. ChemSusChem., 7, 154-161.

Chen W., Yu H., and Liu Y., 2011a. Preparation of millimeterlong cellulose I nanofibers with diameters of $30-80 \mathrm{~nm}$ from bamboo fibers. Carbohydr. Polym., 86, 453-461.

Chen W., Yu H., Liu Y., Chen P., Zhang M., and Hai Y., 2011b. Individualization of cellulose nanofibers from wood using high-intensity ultrasonication combined with chemical pretreatments. Carbohydr. Polym., 83, 1804-1811.

Chen W., Yu H., Liu Y., Hai Y., Zhang M., and Chen P., 2011c. Isolation and characterization of cellulose nanofibers from four plant cellulose fibers using a chemical-ultrasonic process. Cellulose, 18, 433-442.

Clarke A., Prescott T., Khan A., and Olabi A.G., 2010. Causes of breakage and disruption in a homogeniser. Appl. Energy, $87,3680-3690$

Cornell H.J. and Hoveling A.W., 1998. Wheat: chemistry and utilization. CRC Press, Boca Raton, FL, USA.

Cramariuc B., Cramariuc R., Scarlet R., Manea L.R., Lupu I.G., and Cramariuc O., 2013. Fiber diameter in electrospinning process. J. Electroceram., 71, 189-198.

Elleuch M., Bedigian D., Roiseux O., Besbes S., Blecker C., and Attia H., 2011. Dietary fibre and fibre-rich by-products of food processing: Characterisation, technological functionality and commercial applications: A review. Food Chem., 124, 411-421.

Engler C.R. and Robinson C.W., 1981. Disruption of Candida utilis cells in high pressure flow devices. Biotechnol. Bioeng., 23, 765-780.

Foschia M., Peressini D., Sensidoni A., and Brennan C.S., 2013. The effects of dietary fibre addition on the quality of common cereal products. J. Cereal Sci., 8, 216-227.

Gliozzi G., Innorta A., Mancini A., Bortolo R., Perego C., Ricci M., and Cavani F., 2014. $\mathrm{Zr} / \mathrm{P} / \mathrm{O}$ catalyst for the direct acid chemo-hydrolysis of non-pretreated microcrystalline cellulose and softwood sawdust. Appl. Catal., B, Environmental, 145, 24-33.

Gupta P. and Premavalli K.S., 2010. Effect of particle size reduction on physicochemical properties of ashgourd (Benincasa hispida) and radish (Raphanus sativus) fibres. Int. J. Food Sci. Nutr., 61, 18-28.

Hasnaoui N., Wathelet B., and Jimenez-Araujo A., 2014. Valorization of pomegranate peel from 12 cultivars: dietary fibre composition, antioxidant capacity and functional properties. Food Chem., 160, 196-203.

Hassan M.L., Bras J., Hassan E.A., Silard C., and Mauret E., 2014. Enzyme-assisted isolation of microfibrillated cellulose from date palm fruit stalks. Ind. Crops Products, 55, 102-108

Heaney R.P., 2006. Nutrition and chronic disease. Mayo Clinic Proceedings. Mayo Clinic Proc., 81, 297-299. 
Heath L. and Thielemans W., 2010. Cellulose nanowhisker aerogels. Green Chem., 12, 1448.

Hemery Y., Holopainen U., Lampi A.-M., Lehtinen P., Nurmi T., Piironen V., Edelmann M., and Rouau X., 2011. Potential of dry fractionation of wheat bran for the development of food ingredients, part II: Electrostatic separation of particles. J. Cereal Sci., 53, 9-18.

Hemery Y.M., Anson N.M., Havenaar R., Haenen G.R.M.M., Noort M.W.J., and Rouau X., 2010. Dry-fractionation of wheat bran increases the bioaccessibility of phenolic acids in breads made from processed bran fractions. Food Res. Int., 43, 1429-1438.

Huang C.C., Chen Y.F., and Wang C.C., 2010. Effects of micronization on the physico-chemical properties of peels of three root and tuber crops. J. Sci. Food Agric., 90, 759-763.

Ifuku S., Ikuta A., Egusa M., Kaminaka H., Izawa H., Morimoto M., and Saimoto H., 2013. Preparation of highstrength transparent chitosan film reinforced with surface-deacetylated chitin nanofibers. Carbohydr. Polym., 98, 1198-1202.

Kamal-Eldin A., Laerke H.N., Knudsen K.E., Lampi A.M., Piironen V., Adlercreutz H., Katina K., Poutanen K., and Man P., 2009. Physical, microscopic and chemical characterisation of industrial rye and wheat brans from the Nordic countries. Food Nutr. Res., 53.

Lee S.-Y., Chun S.-J., Kang I.-A., and Park J.-Y., 2009. Preparation of cellulose nanofibrils by high-pressure homogenizer and cellulose-based composite films. J. Ind. Eng. Chem., 15, 50-55.

Li M., Wang L.J., Li D., Cheng Y.L., and Adhikari B., 2014. Preparation and characterization of cellulose nanofibers from de-pectinated sugar beet pulp. Carbohydr. Polym., 102, 136-143.

Liu Q., Zhang M., Fang Z.X., and Rong X.H., 2014. Effects of $\mathrm{ZnO}$ nanoparticles and microwave heating on the sterilization and product quality of vacuum packaged Caixin. J. Sci. Food Agric., 94, 2547-2554.

Lu Y., Sun Q., She X., Xia Y., Liu Y., Li J., and Yang D., 2013. Fabrication and characterisation of alpha-chitin nanofibers and highly transparent chitin films by pulsed ultrasonication. Carbohydr. Polym., 98, 1497-1504.

Neves M.A.D., Kimura T., Shimizu N., and Shiiba K., 2006. Production of alcohol by simultaneous saccharification and fermentation of low-grade wheat flour. Brazilian Arch. Biol. Tech., 49, 481-490.

Peng Y., Gardner D.J., and Han Y., 2011. Drying cellulose nanofibrils: in search of a suitable method. Cellulose, 19, 91-102.
Prückler M., Siebenhandl-Ehn S., Apprich S., Höltinger S., Haas C., Schmid E., and Kneifel W., 2014. Wheat branbased biorefinery 1: Composition of wheat bran and strategies of functionalization. LWT - Food Sci. Tech., 56, 211-221.

Rosa N.N., Barron C., Gaiani C., Dufour C., and Micard V., 2013. Ultra-fine grinding increases the antioxidant capacity of wheat bran. J. Cereal Sci., 57, 84-90.

Sangnark A. and Noomhorm A., 2003. Effect of particle sizes on functional properties of dietary fibre prepared from sugarcane bagasse. Food Chem., 80, 221-229.

Tischer P.C.S.F., Sierakowski M.R., Westfahl H., and Tischer C.A., 2010. Nanostructural reorganization of bacterialcellulose by ultrasonic treatment. Biomacromolecules, 11(5), 1217-1224.

Vitaglione P., Napolitano A., and Fogliano V., 2008. Cereal dietary fibre: a natural functional ingredient to deliver phenolic compounds into the gut. Trend Food Sci. Tech., 19, 451-463.

Wang B., Li D., Wang L.-j., Liu Y.-h., and Adhikari B., 2012a. Effect of high-pressure homogenization on microstructure and rheological properties of alkali-treated high-amylose maize starch. J. Food Eng., 113, 61-68.

Wang T., Sun X., Zhou Z., and Chen G., 2012b. Effects of microfluidization process on physicochemical properties of wheat bran. Food Res. Int., 48, 742-747.

Wang C.-C.R., Ciou J.-Y., and Chiang P.-Y., 2009. Effect of micronization on functional properties of the water caltrop (Trapa taiwanensis Nakai) pericarp. Food Chem., 113, 970-974.

Wang T., Sun X., Raddatz J., and Chen G., 2013. Effects of microfluidization on microstructure and physicochemical properties of corn bran. J. Cereal Sci., 58, 355-361.

Wang Y., Wei X., Li J., Wang F., Han D., Wang Q., and Kong L., 2014. Homogeneous isolation of nanocelluloses by controlling the shearing force and pressure in microenvironment. Carbohydr. Polym., 113, 388-393.

Zhu F., Du B., Li R., and Li J., 2014. Effect of micronization technology on physicochemical and antioxidant properties of dietary fiber from buckwheat hulls. Biocata. Agric. Biotech., 3, 4 .

Zhu K., Huang S., Peng W., Qian H., and Zhou H., 2010. Effect of ultrafine grinding on hydration and antioxidant properties of wheat bran dietary fiber. Food Res. Int., 43, 943-948. 